\title{
Smart material using Fiber Bragg Grating transducers and Shape Memory Alloy actuators
}

\author{
L. Rodriguez-Cobo*, A. Quintela, A. Cobo, J.M. Lopez-Higuera \\ Photonics Engineering Group, University of Cantabria, Av los Castros S/N, 39005, \\ Santander, Spain
}

\begin{abstract}
A structure based on a smart material and a PID control loop is presented in this paper. A glass fiber reinforced plastic material is instrumented with Fiber Bragg Gratings (FBG) and Shape Memory Alloy (SMA) actuators. The smart material and the smart structure are both successfully checked by being subjected to different operational conditions at the laboratory. Very good responses are obtained under both slow and quicker varying conditions.
\end{abstract}

Keywords: Smart material, Fiber Bragg Grating, Shape Memory Alloy, PID control, feedback

\section{INTRODUCTION}

The new industrial requirements usually need from very complex structures. There are many examples in which feedback automated systems can upgrade the production with an increment of the reliability such as robotic car factories. The combined use of sensor, smartness and actuator systems enables the development of smart structures, which can respond in a predetermined manner to changes in the environment or in their structural conditions. In particular, new materials with self-response capabilities can be used in lots of scenarios to increase the final performance of the whole structure. Some attempts of fed back structures have been reported using Fiber Bragg Gratings (FBG) [1] such as reducing vibration in a metallic beam with piezoelectric actuators [2] or by modifying a mass position to reduce the vibration of a working structure [3]. Even in robotics field FBG sensors have been reported providing signals to feed a control loop to reduce the vibration of a robot arm [4]. On the other hand Shape Memory Alloy (SMA) actuators have also been applied to smart materials in different fields such as crack suppression on carbon fiber reinforced plastics [5] or to adapt wing geometry [6]. These cases combine both FBG transducers and SMA actuators but it is done in an offline way with no feedback process during the structure operation.

In this work the properties of a smart material actuated by shape memory alloy actuator and feed-backed by Fiber Bragg Grating (FBG) signals under a PID control are shown. The proposed smart material is able to adapt its response to different operational conditions and loads to follow a pre-established target position for specific applications.

\section{STRUCTURE DESIGN}

A simple structure to evaluate the feedback control using SMA actuator is designed based a glass fiber reinforced plastic sheet. FBG transducers are attached and embedded to/in the plastic sheet and a SMA wire actuators are fixed to the other surface of the sheet. A metallic plate is also attached to the center of the structure to provoke a vertical force to the structure allowing the SMA wire to recover its original length. In basic, the structure is designed to obtain a stable vertical position on the metallic plate placed on the top. This position is measured with FBG by quantifying the deformation of the outer surface of the plastic sheet. The actuator wire is designed to reduce its length provoking a vertical displacement of the metallic plate.

*luis.rodrigurez@unican.es; phone: (+34) 942 200877; fax: (+34) 942 200877;

OFS2012 22nd International Conference on Optical Fiber Sensors, edited by

Yanbiao Liao, Wei Jin, David D. Sampson, Ryozo Yamauchi, Youngjoo Chung, Kentaro Nakamura, Yunjiang Rao, Proc. of SPIE Vol. 8421, 8421B1 - (c) 2012 SPIE · CCC code: 0277-786/12/\$18 - doi: 10.1117/12.970622 


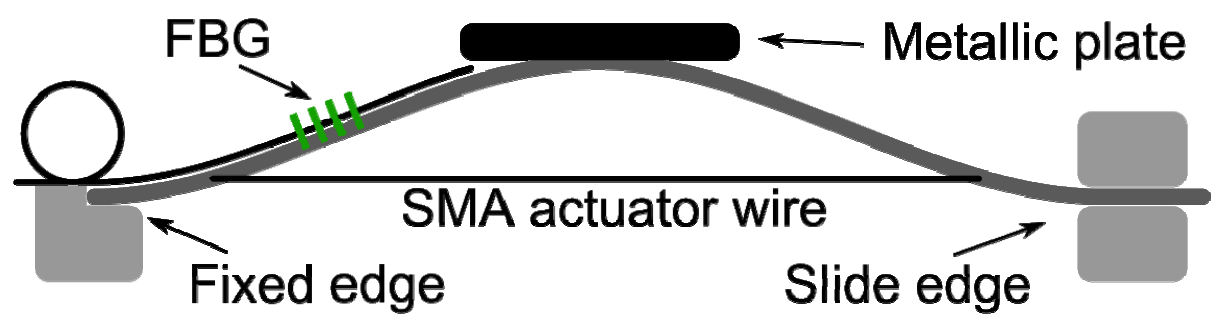

Figure 1. Designed smart structure with an FBG transducer and a SMA actuator wire. Dimensions are in millimeters

\subsection{Shape Memory Alloys}

A Shape Memory Alloy (SMA) is a mixture of different metals that remembers its forged shape (original shape) [7]. The original shape is returned by heating the alloy above a threshold temperature. Basically a SMA changes from one crystallographic state (the martensite state) to other state (the austenite state) as its temperature rises. The movement caused during the transition from the "cool" state (martensite) to the "hot" state is caused by the restructuration of the material. By increasing the temperature above a transition threshold, the original structure is recovered. Once the SMA is cooled again, the original shape is maintained until an external mechanical deformation is applied over it.

For this application, the employed SMA is a nickel-titanium alloy forged in wire shape with a diameter of $0.25 \mathrm{~mm}$ [8]. A typical deformation for this kind of SMA actuators is between $2 \%$ and $5 \%$. For the chosen actuator, the transition temperature is $70^{\circ} \mathrm{C}$, so by heating a deformed actuator above this temperature it will recover its original shape. As shown on the Figure 2, the response during the cooling stage is different of the heating response

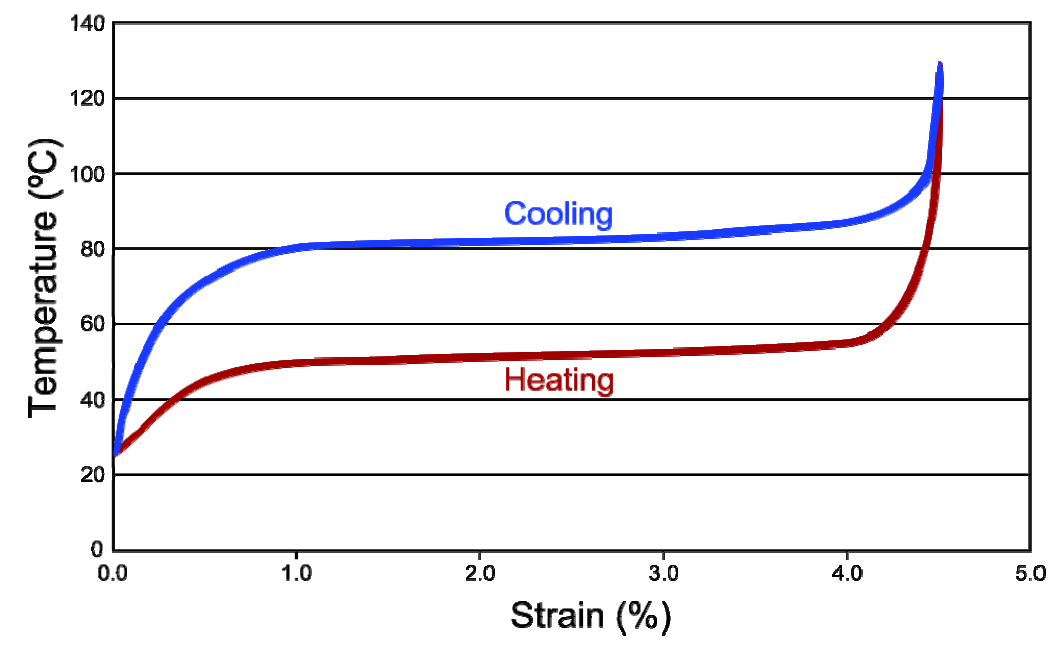

Figure 2. Deformation evolution of the employed SMA wire against the temperature.

The thermal response of the SMA actuators usually is very abrupt causing a very strong deformation in very short time. On the other hand, the cooling process depends on the actuator configuration and its thermal conditions but, usually, is slower than the heating process. Since the actuation movement is caused by the thermal evolution of the SMA, lots of external parameters such as air movement or surrounding materials reflect on the whole system accuracy, making more difficult to get high precision actuators. Once determined data acquisition means (FBG transducer) and the actuator device (SMA wire), to getting a smart material a feedback control to mix up both components are required. For this application a Proportional Integral Derivative (PID) control loop is applied to the SMA actuator using the feedback signal of the FBG transducer. 


\section{EXPERIMENTAL SETUP}

The left end of the proposed structure (shown on the Figure 1) is fixed and the movement of the other one is limited in the height axis and free to the longitudinal axis. When the SMA actuator shorts its length the metallic plate of the top of the structure raises. Due to the pull down force caused by the metallic place, the structure tends to recover its initial position as the actuator is relaxed. The FBG transducer is connected to a Micron Si425-500 interrogation unit and the unit is connected to a PC over Ethernet. The actuator is also connected to the same PC using a custom Pulse Width Modulation (PWM) driver which induces a controllable current to increase the SMA actuator temperature. FBG feedback signal and the actuator driver are linked to a PID control loop implemented over Matlab.

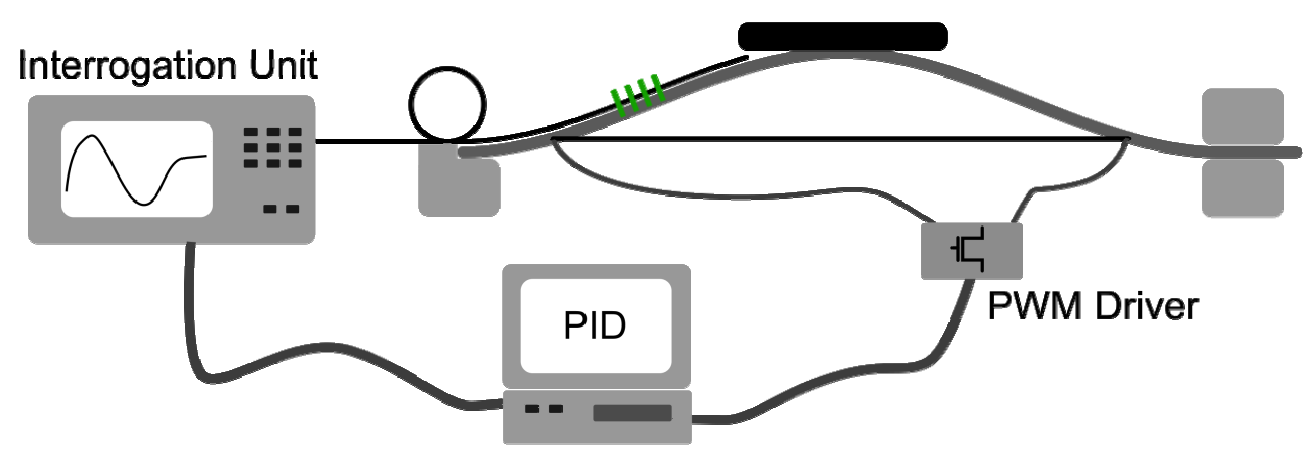

Figure 3. Experimental setup scheme. The FBG transducer of the structure is connected to the interrogation unit and the SMA actuator is connected to the driver. Both elements are linked to a PID control loop.

The sampling frequency of the FBG signals is $2 \mathrm{~Hz}$ and the PWM update period is 2 seconds. In order to get a smother SMA response the current offered to the PWM driver is limited to 1A. The PID control loop gain factors are set empirically. The proposed structure is driven to different target positions measured by the FBG transducer. Once the position of the structure is stable, different extra loads applied to the metallic plate are caused to study the response of the structure to changing conditions.

\section{RESULTS AND DISCUSSION}

In order to analyze the material response, different target positions are fed into de PID control loop maintaining the same structure load. After that, when the material is stable, different loads are applied to analyze the material response. During these test, the room temperature and air flow are not controlled having its influence in the heating and cooling process of the SMA actuator wire. The tuning process of the PID controller is made empirically favoring different aspects as needed such as more stability, faster response or less overshooting.

The response of the smart material to a static situation is shown on the Figure 4 (left). Three different target positions are fed to the PID control (thin line). Control loop is adjusted to generate a slow varying PWM duty cycle (dashed line) to reach the target position from the error evolution (dotted line). The feedback signal from de FBG (wide line) displays the smooth adjust of the structure to the new target.

The smart material response to varying load conditions is also analyzed with a PID faster response configuration, shown on the Figure 4 (right). By removing a 100grams load (dotted circle) the structure reduces the duty cycle of the PWM output signal (dashed line) to maintain the target position. After that, an extra load of 200 grams is added to the structure (solid circle) increasing the value of the output signal. The reaction speed of the structure depends on the PID control parameters offering the possibility of favoring static or dynamic responses. 


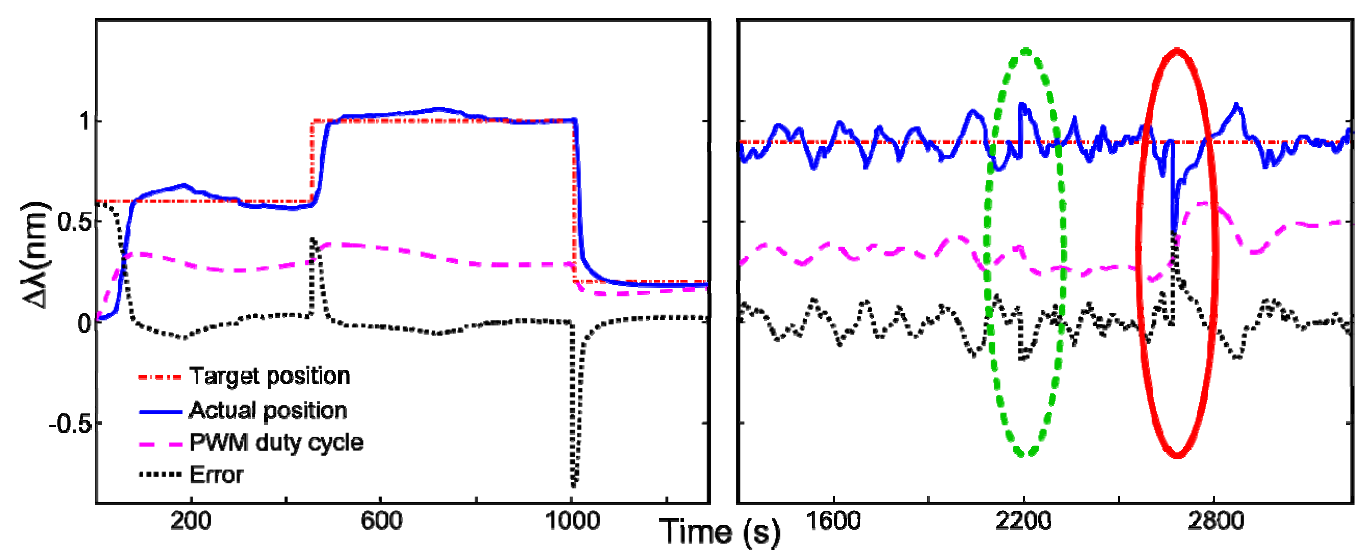

Figure 4. Smart material under 3 stable positions (left) and one target position with variable loads (right).

\section{CONCLUSION}

In this work a new smart structure based on a glass fiber reinforced plastic material instrumented with FBG optical transducers and SMA actuators under a PID control is proposed and tested. The feedback signal coming from the FBGs is fed to the PID loop in order to get a high precision actuator based on the sudden SMA actuator wires. The achieved results prove its self stabilization capabilities even under varying working conditions during long time working situations. The final structure response has to be tuned to favor high precision or high speed by changing the PID control parameters. Both tested situations make up for the sudden response of the SMA actuators.

\section{ACKNOWLEDGMENTS}

This work has been supported by the project TEC2010-20224-C02-02 and grant AP2009-1403. Roberto Perez Sierra is also thanked for his valuable collaboration on the experimental stage.

\section{REFERENCES}

[1] Lopez-Higuera, J. M., Handbook of optical fibre sensing technology: John Wiley \& Sons Inc, 2002.

[2] Chau, K., Moslehi, B., Song, G., and Sethi, V., "Experimental demonstration of fiber Bragg grating strain sensors for structural vibration control," 2004, pp. 753-764.

[3] Cavallo, A., May, C., Minardo, A., Natale, C., Pagliarulo, P., and Pirozzi, S., "Active vibration control by a smart auxiliary mass damper equipped with a fiber Bragg grating sensor," Sensors and Actuators A: Physical, vol. 153, pp. 180-186, 2009.

[4] Chuang, K. C. and Ma, C. C., "Tracking control of a multilayer piezoelectric actuator using a fiber Bragg grating displacement sensor system," Ultrasonics, Ferroelectrics and Frequency Control, IEEE Transactions on, vol. 56, pp. 2036-2049, 2009.

[5] Amano, M., Okabe, Y., and Takeda, N., "Evaluation of Crack Suppression Effect of TiNi SMA Foil Embedded in CFRP Cross-Ply Laminates with Embedded Small-Diameter FBG Sensor," JSME International Journal Series A, vol. 48, pp. 443-450, 2005.

[6] Mieloszyk, M., Skarbek, L., Krawczuk, M., Ostachowicz, W., and Zak, A., "Application of fibre Bragg grating sensors for structural health monitoring of an adaptive wing," Smart Materials and Structures, vol. 20, p. 125014, 2011.

[7] Otsuka, K. and Wayman, C. M., Shape memory materials: Cambridge Univ Pr, 1999.

[8] http://www.dynalloy.com/TechDataWire.php 